\title{
Highly conductive and transparent metal-organic frameworks thin film
}

\author{
Zhuoyi Li, Yi Guo, Wen Ying, Danke Chen, Xiaobin Wang, Xu Ma, Xing Zhao, Zheng Deng and \\ Xinsheng Peng ${ }^{*}$
}

Energy crisis has aroused global concern and needs to be solved immediately. A series of serious environmental problems caused by the unlimited use of traditional fossil fuels like petroleum and charcoal, such as rising sea levels and melting glaciers, have dangerously threatened human destiny [1]. It is an effective choice to look for new energy sources to replace the limited fossil fuel reserves. Among these new energy sources, solar energy stands out and attracts tremendous attention because of its environmental friendliness, stability, renewability, and universality [2-5]. Transparent and conductive electrode is the vital part in photoelectric conversion devices [6]. Indium tin oxide (ITO) is the most popular transparent and conductive electrode material at present [7]. However, the limited reserves of indium, a component in ITO has resulted in the high price of ITO electrode, restricting its wide application.

As a new kind of porous crystalline material, metalorganic frameworks (MOFs) have become a rapidly developing research area. MOFs can be constructed according to the desired properties by selecting specific central metal ions and different functional organic ligands. This structure-adjustable feature makes MOF a material that can perform multiple functions. In addition, MOFs have high specific surface area, high porosity and accessible metal sites and so on [8-14]. These merits make MOFs be widely used in gas separation and storage [15-17], catalysis [18-24], drug delivery [25,26], sensing [27-29], supercapacitor [30] and so on.

Nevertheless, research on MOFs applied as transparent electrode for photo-electrochemical catalyst device, which requires high porosity, high surface area, high conductivity and high light transmittance, has encountered great challenges since most MOFs are either opaque or insulated. In fact, only a few MOFs have high light transmittance and most of MOFs have poor conductivity, often lower than $10^{-10} \mathrm{~S} \mathrm{~cm}^{-1}$ [31]. In general, there are two ways to increase the conductivity of MOFs. One is to select suitable organic ligand and central metal ion to improve its conductivity through the interaction between them [32]. However, it needs extremely expensive organic ligand, complicated reaction conditions and long reaction time $[33,34]$. The other is to prepare a composite material by incorporating the functional particles into highly porous MOFs [35,36]. The key point is the functional particle size must match well with the entrance size of MOFs. However, it is hard to obtain transparent and conductive MOFs thin film. Poly(3,4-ethylenedioxythiophene):poly(styrenesulfonic acid) (PEDOT:PSS) is an ideal material to modify MOFs so as to prepare functionalized composite materials since it is a conductive polymer material with high conductivity and good light transmittance, which has been widely used in organic solar cells, light-emitting diodes and supercapacitors [37-41]. Recently, highly conductive and transparent PEDOT:PSS thin films have been prepared for various applications [6,30,39-41], while the incorporation of PEDOT:PSS into MOFs matrix is scarcely reported.

In this work, we report a simple strategy to prepare a highly conductive and transparent PEDOT:PSS@ZIF-8 (PPZ) composite thin film by incorporating highly conductive PEDOT:PSS into porous and transparent ZIF-8 via a solid conversion process as we described elsewhere [42-44]. The as-prepared PPZ thin film avoids the poor electron conductivity of ZIF- 8 and possesses the merits originated from both transparent ZIF-8 and conductive PEDOT:PSS. To be specific, the PPZ thin film maintains good transmittance, high Brunauer-Emmett-Teller (BET) surface area as well as regular and stable microstructure inherited from ZIF-8. Meanwhile, the PPZ thin film

State Key Laboratory of Silicon Materials, School of Materials Science and Engineering, Zhejiang University, Hangzhou 310027, China

${ }^{*}$ Corresponding author (email: pengxinsheng@zju.edu.cn) 
shows high conductivity and good transmittance. The properties of the PPZ thin film are further remarkably improved by dimethyl sulfoxide (DMSO) modification. The DMSO-modified PPZ thin films show better conductivity and transmittance than the unmodified one. Moreover, the PPZ thin film containing only $20 \mathrm{wt} \%$ of PEDOT:PSS displays high conductivity and good transmittance, which are close to those of pure PEDOT:PSS thin film.

It mainly contains three steps to prepare a PPZ thin film (Scheme 1). First, the PEDOT:PSS/ZHNs mixed solution was prepared by simply mixing the positively charged zinc hydroxide nanostrands (ZHNs) with negatively charged PEDOT:PSS solution. Then a PEDOT:PSS/ ZHNs composite thin film (Fig. 1a) was obtained by filtering the PEDOT:PSS/ZHNs mixed solution on a porous polycarbonate (PC) membrane. The composite nanofibrous structures are clearly observed, which means the addition of PEDOT:PSS does not damage the linear nanofibrous structure of ZHNs. Finally, a PPZ thin film was obtained by transferring the PEDOT:PSS/ZHNs composite thin film onto a piece of quartz glass, and immersing it into 2-methylimidazole (2-MI) water-ethanol (water/ ethanol volume ratio 4:1) solution at room temperature for $24 \mathrm{~h}$. Fig. 1 shows a continuous and well intergrown crystal thin film is formed. PPZs with different amounts of PEDOT:PSS (PPZ with 1, 5, 10, 15, 20, 25 and $30 \mathrm{wt} \%$ PEDOT:PSS was denoted as PPZ-1, PPZ-5, PPZ-10, PPZ15, PPZ-20, PPZ-25, and PPZ-30, respectively) were synthesized by varying the volume ratio of ZHNs to PEDOT:PSS (see details in the Supplementary information). Fig. 1 and Fig. S1 show that all the PPZ thin films with different amounts of PEDOT:PSS are well intergrown and continuous.
Several analysis methods were used to confirm the successful confinement of PEDOT:PSS in ZIF-8 thin film. The energy-dispersive X-ray spectroscopy (EDXS) mapping analysis performed on the cross-section of PPZ-20 (Fig. S2e) shows that $S$ originating from PEDOT:PSS is uniformly distributed in the PPZ thin film. Besides, in the Fourier transform infrared (FTIR) spectroscopy of PPZ (Fig. $2 \mathrm{~b}$ and Fig. S3a), the $\mathrm{C}=\mathrm{C}$ and $\mathrm{C}-\mathrm{C}$ stretching of the quinoidal group in the thiophene rings and the sulfonic acid groups of PSS are observed at ca. 1536 and $1330 \mathrm{~cm}^{-1}$, respectively. The vibrations at ca. $1215 \mathrm{~cm}^{-1}$ are assigned to the $\mathrm{C}-\mathrm{O}-\mathrm{C}$ bond stretching in ethylene dioxy group. The peaks at ca. 951 and $615 \mathrm{~cm}^{-1}$ originate from the $\mathrm{C}-\mathrm{S}$ bond stretching in the thiophene ring $[45,46]$. Moreover, from the X-ray photoelectron spectroscopy (XPS) of the PPZ thin film (Fig. 2c and Fig. S3b), the peaks with binding energies of $162-172 \mathrm{eV}$ are ascribed to sulfur originating from PEDOT:PSS [47] while no peak of sulfur is observed in the XPS spectra of ZIF-8. Comparing the XPS spectra of the PPZ thin film conducted with surface analysis method and depth dissection method, obvious peaks of the sulfur element are observed. These indicate PEDOT:PSS is encapsulated in ZIF-8 thin film while partially exposing out of ZIF-8 thin film. Besides the analysis methods mentioned above which directly confirm the well confinement of PEDOT:PSS in the PPZ thin film, it also can be proved indirectly by analyzing the porosity of ZIF- 8 and the PPZ thin films. The pore size distributions of pristine ZIF-8, PPZ-10, PPZ-20 and PPZ-30 are shown in Fig. S3d. The $\mathrm{N}_{2}$ sorption isotherms of ZIF-8, PPZ-5, PPZ-10, PPZ-20, and PPZ-30 at $77 \mathrm{~K}$ are shown in Fig. S3c, all of which display approximately type I isotherms. The BET surface areas of ZIF-8, PPZ-5, PPZ-10, PPZ-20 and PPZ-30 (Fig. 2d) are

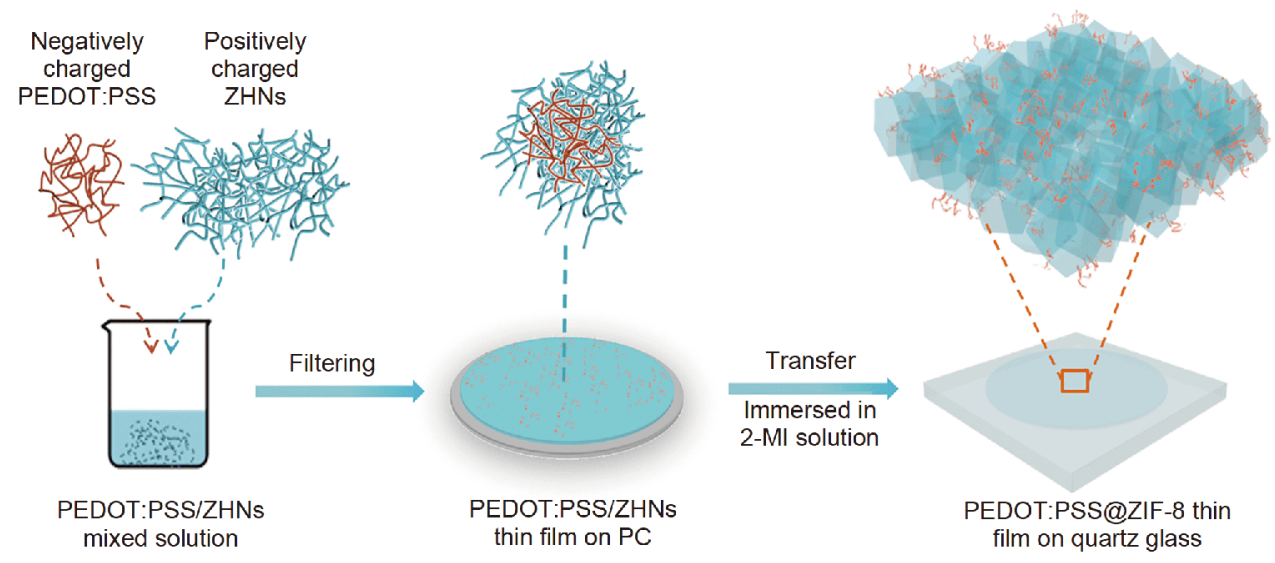

Scheme 1 Illustration of the preparation process of PEDOT:PSS@ZIF-8 thin film. 

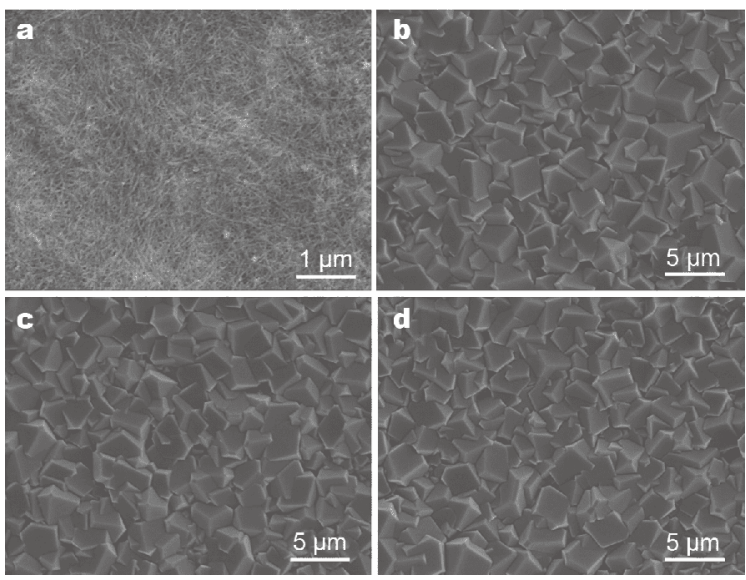

Figure 1 Surface SEM images of PEDOT:PSS/ZHNs (a), ZIF-8 thin film (b), the PPZ-20 thin film (c), the PPZ-20 thin film after DMSO modification (d).

$1636.79,1604.61,1360.10,1266.11 \mathrm{~m}^{2} \mathrm{~g}^{-1}$, respectively. Apparently, the BET surface area of the PPZ thin film decreases when more PEDOT:PSS is added during the synthesis process, indicating more PEDOT:PSS is included into the PPZ thin film. Even so, the BET surface area of the PPZ thin films are still high.

In the X-ray diffraction (XRD) patterns of ZIF-8 and the PPZ thin films (PPZ-5, PPZ-10, PPZ-20 and PPZ-30) (Fig. 2a), it is obvious that the patterns of the PPZ thin films match perfectly with that of ZIF-8, implying the inclusion of amorphous conductive polymer, PEDOT: PSS, does not alter the crystalline phase of ZIF- 8 . The preservation of the structures of ZIF- 8 after the incorporation of PEDOT:PSS can be proved by the scanning electron microscopy (SEM) images of ZIF- 8 and the PPZ thin films. The surface SEM images (Figs 1b, S1a, S1c, S1e) of ZIF-8, PPZ-5, PPZ-10, PPZ-20 and PPZ-30 thin films show that the shape and size of crystals are uniform, with their sizes ranging from 2 to $3 \mu \mathrm{m}$ and all the thin films are intergrown and compact. Similarly, the thicknesses of all the PPZ thin films (PPZ-5, PPZ-10, PPZ-20 and PPZ-30) (Figs S1b, S1d, S2a and S1f, respectively) are approximately $1 \mu \mathrm{m}$. Consequently, it is clear that the inclusion of PEDOT:PSS has negligible effects on the structure of ZIF-8.

The conductivity of ZIF- 8 and the PPZ thin films (PPZ1, PPZ-5, PPZ-10, PPZ-15, PPZ-20, PPZ-25 and PPZ-30) (Fig. 3a) were obtained by a two-probe current-voltage ( $I$ $V)$ method with two Ag electrodes evaporated on the surface of the thin films (Fig. S4) and a four-probe conductivity measurement method, respectively. The conductivity of the PPZ thin film obtained by the four-probe
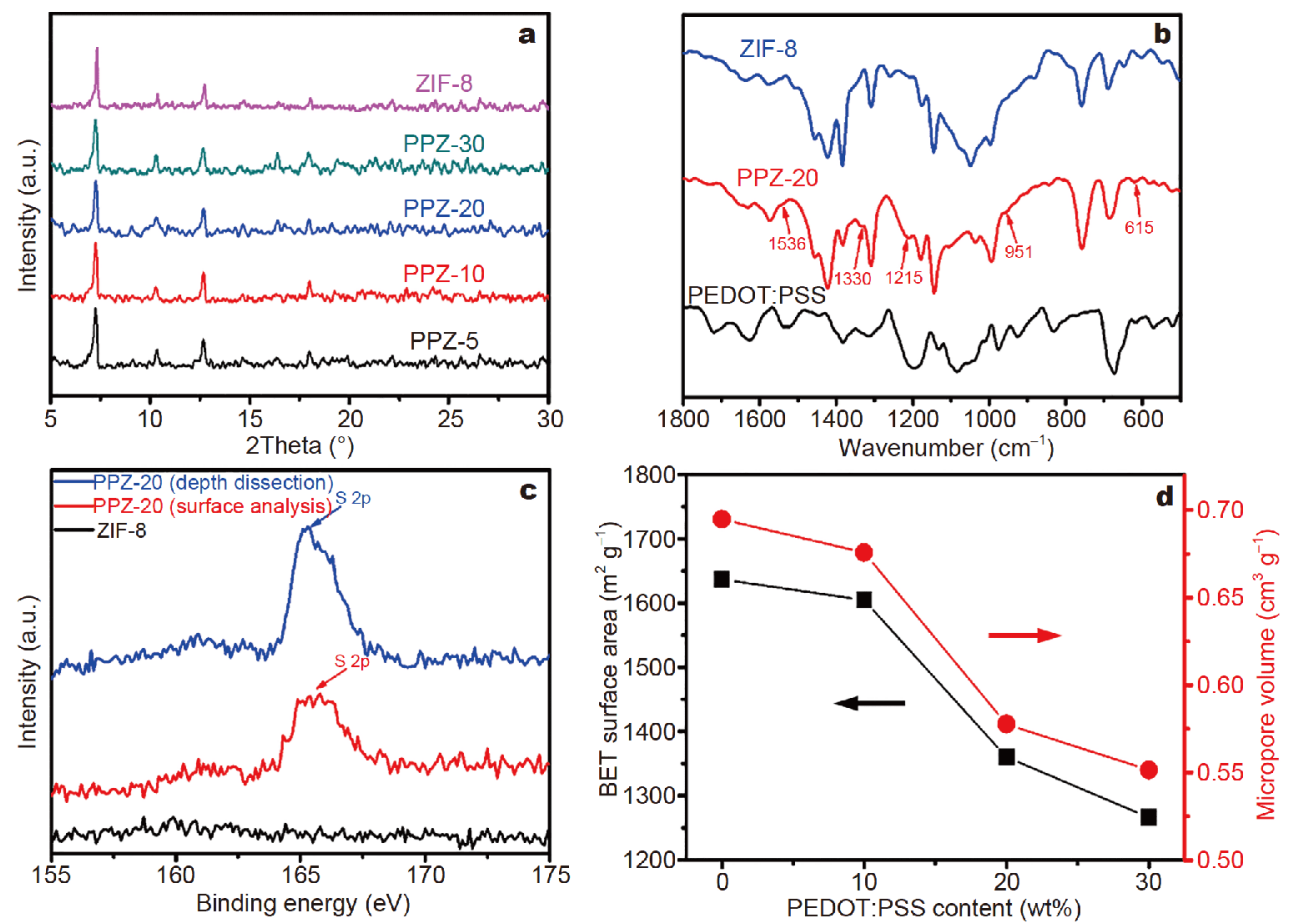

Figure 2 (a) XRD patterns of ZIF-8 and the PPZ thin films. (b) FTIR of ZIF-8, PEDOT:PSS and the PPZ thin films. (c) XPS of ZIF-8 and the PPZ thin films. (d) BET surface areas and micropore volumes of ZIF-8 and the PPZ thin films. 

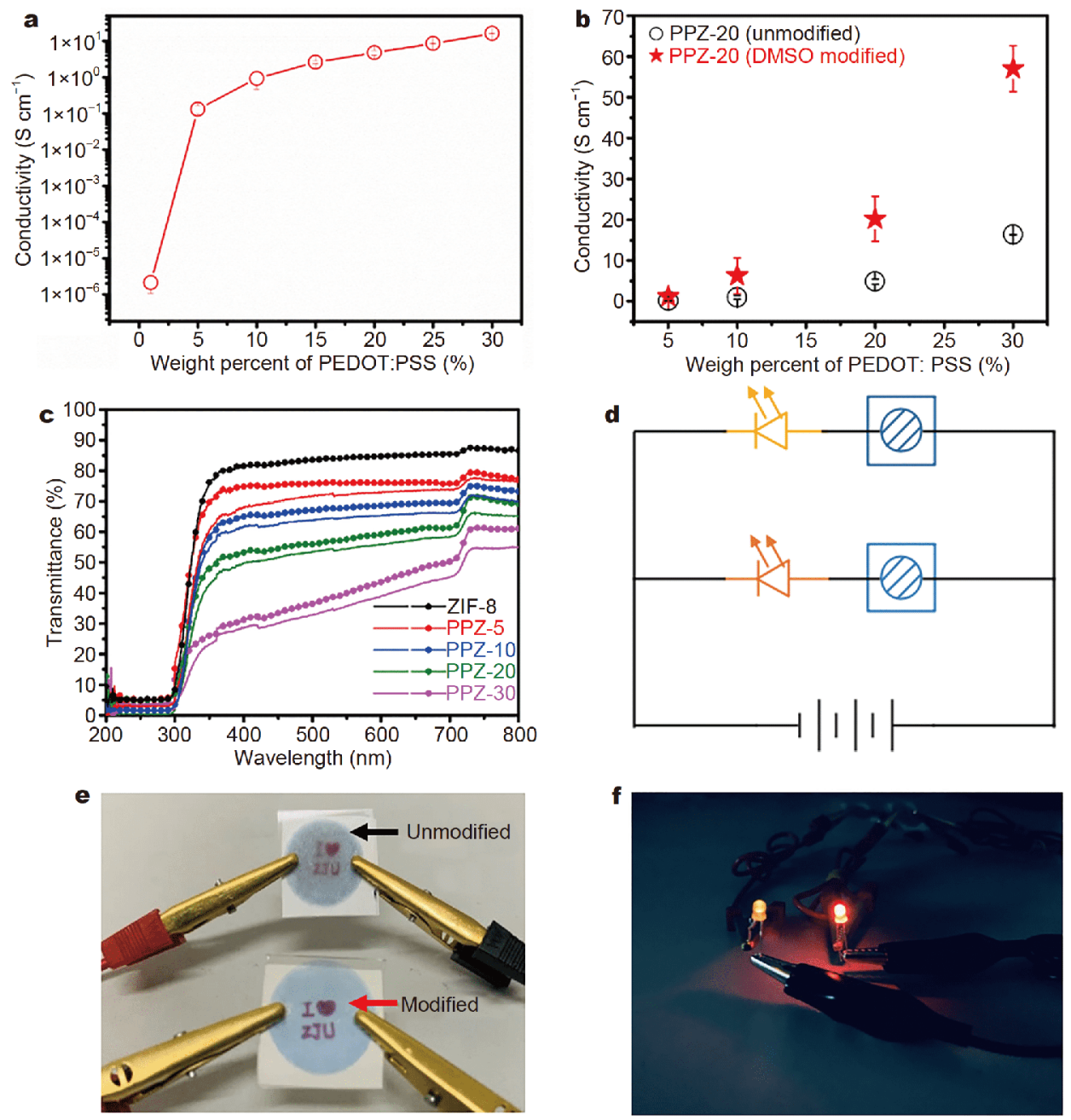

Figure 3 (a) Conductivity of the PPZ thin films (with 1, 5, 10, 15, 20, 25, and 30 wt\% PEDOT:PSS, respectively). (b) Conductivity of the PPZ thin films before and after DMSO modification. (c) Light transmittance of ZIF-8 and the PPZ thin films before (lines without symbols) and after (lines with symbols) DMSO modification. (d) Circuit diagram of a closed loop. (e, f) Photographs of the PPZ thin film (with and without modification) in a closed loop with a power supply $(6 \mathrm{~V})$ lighting a red LED device.

conductivity measurement is slightly higher than that of the two-probe process since the former eliminates the contact resistance. The conductivity of the PPZ thin film increases with the increase of PEDOT:PSS amount in the PPZ thin film. The average conductivity of PPZ-30 is $16.37 \mathrm{~S} \mathrm{~cm}^{-1}$, which is the highest conductivity among all the PPZ thin films, while the pure ZIF-8 thin film is nearly insulative. The greatly enhanced conductivities of the PPZ thin films are attributed to the highly conductive and evenly distributed PEDOT:PSS, which works not only as a conductive additive but also as a binder, providing the pathway for electrons to transport and connecting with ZIF-8 to form an entire conductive network. Therefore, PEDOT:PSS plays an indispensible and crucial role in improving the conductivity of the PPZ thin film.

Given the good conductivity of the PPZ thin film due to the inclusion of PEDOT:PSS, the high light transmittance of ZIF-8 thin film is unavoidably sacrificed. The light transmittance of ZIF-8 in the wavelength range from 350 to $700 \mathrm{~nm}$ is about $85 \%$. But the light transmittance of the PPZ thin film decreases significantly due to the incorporation of PEDOT:PSS. This is because (i) the light transmittance of PEDOT:PSS is no better than that of ZIF-8; (ii) the PEDOT:PSS in the PPZ thin film is not as compact and tight as in the pure PEDOT:PSS thin film which has good light transmittance. To solve this intractable problem, we utilized DMSO to modify the PPZ thin film. The light transmittance of all modified PPZ 
thin films increases as expected while the light transmittance of ZIF-8 shows no obvious difference (Fig. 3c). To further evaluate the influence of DMSO on the PPZ thin film, we measured the conductivity of PPZ thin films modified with DMSO using two-probe current-voltage $(I-V)$ method. Compared with the original PPZ thin film, the modified PPZ thin film shows a great improvement of conductivity (Fig. 3b). To be specific, the conductivity of the PPZ-30 thin film increases from 16.37 to $57.03 \mathrm{~S} \mathrm{~cm}^{-1}$. The enhancement of both conductivity and light transmittance of the PPZ thin film are attributed to DMSO. DMSO changes the connection mode and interaction force between PEDOT and PSS, which leads to phase separation of PEDOT and PSS by charge screening. After phase separation, the conductive PEDOT chains are more compact with bigger contact area. Besides, they stay far away from the insulative PSS chain, electrically facilitating better electrical connection [48-50]. Moreover, from the SEM image of the modified PPZ thin films (Fig. 1d), we conclude that DMSO does not affect the microstructure of the PPZ thin film. DMSO modification improves the conductivity and light transmittance of the PPZ thin film without damaging its shape and structure.

Considering that the modified PPZ thin film exhibits both good light transmittance and conductivity, we prospected that it might be a good transparent electrode. As a proof-of-concept, we built a closed loop using the PPZ-20 thin film as a conductive electrode (Fig. 3d and Fig. S5). When simultaneously working at the same closed loop, the light transmittance and conductivity of the PPZ-20 thin film with/without DMSO modification show a big dissimilarity. Apparently, although under the same applied voltage $(6 \mathrm{~V})$, the light-emitting diodes which is in series with the modified PPZ-20 thin film is brighter than the one with the same specification but in series with the unmodified PPZ-20 thin film (Fig. 3f), implying the current flowing through the former lightemitting diodes is bigger than the latter. Namely, the modified PPZ-20 thin film has a higher electrical conductivity than the unmodified one. As for light transmittance differences between these two PPZ-20 thin films, we wrote down the same text with the same size and color on a paper whose size is identical with the quartz glass. The text under the DMSO modified PPZ-20 thin film is clearer than the one under the unmodified PPZ-20 thin film (Fig. 3e), showing better light transmittance of the modified PPZ-20 thin film. These experimental results directly display that the characteristics of the DMSO modified PPZ thin film will satisfy the requirements for being used for transparent electrodes.
Further research is still needed to improve the performance.

In conclusion, we prepared a highly conductive and transparent MOFs thin film PPZ using a solid confinement method by incorporating the highly conductive PEDOT:PSS into ZIF-8 thin film. To our knowledge, this is the first time that MOFs thin film with both good conductivity and light transmittance was prepared. As expected, the as-prepared PPZ thin film inherits the genius of both ZIF-8 and PEDOT:PSS, showing excellent properties. On one hand, the PPZ thin film perfectly retains the microstructure and crystalline phase of ZIF-8, displaying uniform crystal size and regular pore size. On the other hand, PEDOT:PSS included in the PPZ thin film works as both conductive additive and binder, which tremendously enhances the conductivity of the PPZ thin film. And the more PEDOT:PSS the PPZ thin film contains, the higher conductivity the PPZ thin film has. Surprisingly, the conductivity of PPZ-30 is $16.37 \mathrm{~S} \mathrm{~cm}^{-1}$, while ZIF-8 is insulative. Nevertheless, the inclusion of PEDOT:PSS into ZIF-8 thin film unavoidably occupies some space of ZIF-8 thin film, namely, sacrificing some BET surface area and pore volume of ZIF-8, but the PPZ thin film still retains relatively high BET surface area; besides, the high light transmittance of ZIF- 8 thin film is partly sacrificed due to the inclusion of PEDOT:PSS. Referring to the pioneer's work, we introduced DMSO to modify the PPZ thin film to make up for the decrement of light transmittance. To our expectation, the light transmittance of the modified PPZ thin film is increased and the conductivity of PPZ is further enhanced, the highest conductivity of the DMSO modified PPZ thin films is $57.03 \mathrm{~S} \mathrm{~cm}^{-1}, 3.5$ times as that of the PPZ thin films without modification. The modified additive, DMSO, contributes to the phase separation of PEDOT and PSS, resulting in the enhancement of conductivity and transmittance of the PPZ thin film. Given the good conductivity and transmittance, the DMSO modified PPZ thin film shows great potential in its application as transparent electrode, such as photo-electrochemical catalyst device, which will expand the application of MOFs. Moreover, the method to prepare the conductive and transparent PPZ thin film provides a new avenue for synthesizing conductive and transparent MOFs materials.

\section{Received 4 March 2019; accepted 18 April 2019; published online 07 May 2019}

1 Armaroli N, Balzani V. The future of energy supply: Challenges and opportunities. Angew Chem Int Ed, 2007, 46: 52-66

2 Kudo A, Yugo M. Heterogeneous photocatalyst materials for water 
splitting. Catal Surveys Asia, 2003, 7: 31-38

3 Lewis NS. Toward cost-effective solar energy use. Science, 2007, 315: 798-801

4 Walter MG, Warren EL, McKone JR, et al. Solar water splitting cells. Chem Rev, 2010, 110: 6446-6473

5 Zhang $\mathrm{P}$, Wang $\mathrm{T}$, Chang $\mathrm{X}$, et al. Effective charge carrier utilization in photocatalytic conversions. Acc Chem Res, 2016, 49: 911921

6 Li D, Lai WY, Zhang YZ, et al. Printable transparent conductive films for flexible electronics. Adv Mater, 2018, 30: 1704738

7 Das MC, Xiang S, Zhang Z, et al. Functional mixed metal-organic frameworks with metalloligands. Angew Chem Int Ed, 2011, 50: 10510-10520

8 Gather MC, Köhnen A, Meerholz K. White organic light-emitting diodes. Adv Mater, 2011, 23: 233-248

9 Li H, Eddaoudi M, O'Keeffe M, et al. Design and synthesis of an exceptionally stable and highly porous metal-organic framework. Nature, 1999, 402: 276-279

10 James SL. Metal-organic frameworks. Chem Soc Rev, 2003, 32: 276-288

11 Jiang HL, Xu Q. Porous metal-organic frameworks as platforms for functional applications. Chem Commun, 2011, 47: 3351-3370

12 Zhou HC, Long JR, Yaghi OM. Introduction to metal-organic frameworks. Chem Rev, 2012, 112: 673-674

13 Guo Y, Peng X. Mass transport through metal organic framework membranes. Sci China Mater, 2019, 62: 25-42

$14 \mathrm{Xu} \mathrm{X}, \mathrm{Lu} \mathrm{Y}$, Yang Y, et al. Tuning the growth of metal-organic framework nanocrystals by using polyoxometalates as coordination modulators. Sci China Mater, 2015, 58: 370-377

$15 \mathrm{Wu} \mathrm{H}$, Gong Q, Olson DH, et al. Commensurate adsorption of hydrocarbons and alcohols in microporous metal organic frameworks. Chem Rev, 2012, 112: 836-868

16 Li JR, Sculley J, Zhou HC. Metal-organic frameworks for separations. Chem Rev, 2012, 112: 869-932

17 Sumida K, Rogow DL, Mason JA, et al. Carbon dioxide capture in metal-organic frameworks. Chem Rev, 2012, 112: 724-781

18 Lee JY, Farha OK, Roberts J, et al. Metal-organic framework materials as catalysts. Chem Soc Rev, 2009, 38: 1450-1459

19 Yoon M, Srirambalaji R, Kim K. Homochiral metal-organic frameworks for asymmetric heterogeneous catalysis. Chem Rev, 2012, 112: 1196-1231

20 Wu Q, Liang J, Yi JD, et al. Porous nitrogen/halogen dual-doped nanocarbons derived from imidazolium functionalized cationic metal-organic frameworks for highly efficient oxygen reduction reaction. Sci China Mater, 2019, 62: 671-680

21 Wu S, Zhu Y, Huo Y, et al. Bimetallic organic frameworks derived $\mathrm{CuNi} /$ carbon nanocomposites as efficient electrocatalysts for oxygen reduction reaction. Sci China Mater, 2017, 60: 654-663

22 Liu X, Tang B, Long J, et al. The development of MOFs-based nanomaterials in heterogeneous organocatalysis. Sci Bull, 2018, 63: $502-524$

23 Zhang TR. Tunable chiral metal organic frameworks for visible light-driven asymmetric catalysis. Acta Phys-Chim Sin, 2018, 34: 235-236

24 Shang L, Yu H, Huang X, et al. Well-dispersed ZIF-derived Co,Nco-doped carbon nanoframes through mesoporous-silica-protected calcination as efficient oxygen reduction electrocatalysts. Adv Mater, 2016, 28: 1668-1674

25 Vallet-Regí M, Balas F, Arcos D. Mesoporous materials for drug delivery. Angew Chem Int Ed, 2007, 46: 7548-7558
26 Horcajada P, Chalati T, Serre C, et al. Porous metal-organicframework nanoscale carriers as a potential platform for drug delivery and imaging. Nat Mater, 2010, 9: 172-178

27 Jiang HL, Tatsu Y, Lu ZH, et al. Non-, micro-, and mesoporous metal-organic framework isomers: reversible transformation, fluorescence sensing, and large molecule separation. J Am Chem Soc, 2010, 132: 5586-5587

28 Kreno LE, Leong K, Farha OK, et al. Metal-organic framework materials as chemical sensors. Chem Rev, 2011, 112: 1105-1125

29 Zhou X, Cheng J, Li L, et al. A europium(III) metal-organic framework as ratiometric turn-on luminescent sensor for $\mathrm{Al}^{3+}$ ions. Sci China Mater, 2018, 61: 752-757

30 Zhang YZ, Cheng T, Wang Y, et al. A simple approach to boost capacitance: flexible supercapacitors based on manganese oxides@MOFs via chemically induced in situ self-transformation. Adv Mater, 2016, 28: 5242-5248

31 Sun L, Campbell MG, Dincă M. Electrically conductive porous metal-organic frameworks. Angew Chem Int Ed, 2016, 55: 35663579

32 Hmadeh M, Lu Z, Liu Z, et al. New porous crystals of extended metal-catecholates. Chem Mater, 2012, 24: 3511-3513

33 Sun L, Hendon CH, Minier MA, et al. Million-fold electrical conductivity enhancement in $\mathrm{Fe}_{2}(\mathrm{DEBDC})$ versus $\mathrm{Mn}_{2}$ (DEBDC) ( $\mathrm{E}$ = S, O). J Am Chem Soc, 2015, 137: 6164-6167

34 Dong RH, Pfeffermann M, Liang H, et al. Large-area, free-standing, two-dimensional supramolecular polymer single-layer sheets for highly efficient electrocatalytic hydrogen evolution. Angew Chem Int Ed, 2015, 54: 12058-12063

35 Talin AA, Centrone A, Ford AC, et al. Tunable electrical conductivity in metal-organic framework thin-film devices. Science, 2014, 343: 66-69

36 Dhara B, Nagarkar SS, Kumar J, et al. Increase in electrical conductivity of MOF to billion-fold upon filling the nanochannels with conducting polymer. J Phys Chem Lett, 2016, 7: 2945-2950

37 Aasmundtveit KE, Samuelsen EJ, Pettersson LAA, et al. Structure of thin films of poly(3,4-ethylenedioxythiophene). Synth Met, 1999, 101: 561-564

38 Martin DC, Wu J, Shaw CM, et al. The Morphology of poly(3,4ethylenedioxythiophene). Polymer Rev, 2010, 50: 340-384

39 Cheng T, Zhang YZ, Yi JP, et al. Inkjet-printed flexible, transparent and aesthetic energy storage devices based on PEDOT:PSS/Ag grid electrodes. J Mater Chem A, 2016, 4: 13754-13763

40 Cheng T, Zhang YZ, Zhang JD, et al. High-performance freestanding PEDOT:PSS electrodes for flexible and transparent allsolid-state supercapacitors. J Mater Chem A, 2016, 4: 10493-10499

41 Zhou L, Yu M, Chen X, et al. Screen-printed poly(3,4-ethylenedioxythiophene):poly(styrenesulfonate) grids as ITO-free anodes for flexible organic light-emitting diodes. Adv Funct Mater, 2018, 28: 1705955

42 Mao Y, Li J, Cao W, et al. General incorporation of diverse components inside metal-organic framework thin films at room temperature. Nat Commun, 2014, 5: 5532

43 Guo Y, Ying Y, Mao Y, et al. Polystyrene sulfonate threaded through a metal-organic framework membrane for fast and selective lithium-ion separation. Angew Chem Int Ed, 2016, 55: 15120-15124

44 Guo Y, Jiang Z, Ying W, et al. A DNA-threaded ZIF-8 membrane with high proton conductivity and low methanol permeability. Adv Mater, 2017, 30: 1705155

45 Xiao Y, Lin JY, Tai SY, et al. Pulse electropolymerization of high 
performance PEDOT/MWCNT counter electrodes for Pt-free dyesensitized solar cells. J Mater Chem, 2012, 22: 19919

46 Sonawane NB, Ahire RR, Gurav KV, et al. PEDOT:PSS shell on CdS nanowires: Room temperature LPG sensor. J Alloys Compd, 2014, 592: 1-5

47 Song W, Fan X, Xu B, et al. All-solution-processed metal-oxidefree flexible organic solar cells with over $10 \%$ efficiency. Adv Mater, 2018, 30: 1800075

48 Wijeratne K, Ail U, Brooke R, et al. Bulk electronic transport impacts on electron transfer at conducting polymer electrodeelectrolyte interfaces. Proc Natl Acad Sci USA, 2018, 115: 1189911904

49 Shi H, Liu C, Jiang Q, et al. Effective approaches to improve the electrical conductivity of PEDOT:PSS: a review. Adv Electron Mater, 2015, 1: 1500017

50 Yildirim E, Wu G, Yong X, et al. A theoretical mechanistic study on electrical conductivity enhancement of DMSO treated PEDOT: PSS. J Mater Chem C, 2018, 6: 5122-5131

Acknowledgements This work was supported by the Major R\&D Plan of Zhejiang Natural Science Foundation (LD18E020001), the National Key Research and Development Program (2016YFA0200204), the Key Program of National Natural Science Foundation (51632008), the National Natural Science Foundation of China (21671171 and 21875212), and the National Basic Research Program of China (973 Program, 2015CB655302).

Author contributions Peng X supervised the project. Li Z designed and performed the experiments, analyzed the results and wrote the manuscript. Guo Y, Wang X, Ma X and Zhao X assisted in the device fabrication and measurement. Deng Z, Ying W and Chen D contributed to the data analysis. All authors contributed to the general discussion.

Conflict of interest The authors declare that they have no conflict of interest.

Supplementary information Experimental details and supporting data are available in the online version of the paper.

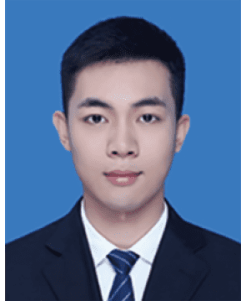

Zhuoyi Li received his bachelor degree (2017) in the School of Metallurgy and Environment at Central South University. He is currently pursuing his PhD degree in the School of Materials Science and Engineering at Zhejiang University under the supervision of Prof. Xinsheng Peng. His present research interests mainly focus on the design and synthesis of electrically conductive metal-organic frameworks (MOF) membranes for energy conversion devices.

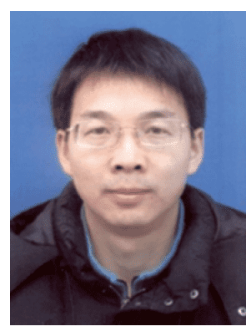

Xinsheng Peng received his PhD in 2003 at the Institute of Solid State Physics, Chinese Academy of Sciences. He became a full professor at the School of Materials Science and Engineering at Zhejiang University in 2010. His research interests mainly focus on the design and synthesis of functional membranes and controlled mass transportation in energy and environmental science.

\section{一种高导电性透明金属有机骨架物薄膜}

李卓异, 郭弯, 应文, 陈丹科, 王小涁, 马旭, 赵星, 邓正, 彭新生 ${ }^{*}$

摘要 金属有机框架(MOFs) 以其优良的特性和广泛的应用而备受关 注. 然而, 当应用于透明导电薄膜器件时, 其导电性差且透光率低. 因 此提高MOF基材料的导电性和透光性成为最棘手的难题. 本文报道 了一种将高导电性PEDOT:PSS引入透明金属有机骨架ZIF-8薄膜中并 成功合成具有高导电性、高透光性的PEDOT:PSS@ZIF-8 (PPZ)金属 有机骨架复合薄膜的有效方法. 相对于近乎绝缘的纯ZIF-8薄膜, 我 们制备的PPZ薄膜的最高导电率高达 $16.37 \mathrm{~S} \mathrm{~cm}^{-1}$. 加入PEDOT:PSS 后, ZIF-8薄膜透光率降低, 因此我们利用二甲亚砜对PPZ薄膜进一步 改性. 改性后的PPZ薄膜比原PPZ薄膜具有更高的导电性和更好的透 光性, 其最高导电率为 $57.03 \mathrm{~S} \mathrm{~cm}^{-1}$, 是未改性PPZ薄膜的 3.5 倍. PPZ薄 膜展现了其应用于透明导电器件的巨大潜力, 并为制备具有高导电 性和良好透光性的MOF基材料提供了新途径. 